\title{
A Rare Antitiy Extrarenal Wilms Tumor: A Case Report
}

\section{Sezen Karaca Ozkisacik*, Ali Onur Erdem and Mesut Yazici \\ Department of Pediatric Surgery, Adnan Menderes University, Turkey}

*Corresponding author: Sezen Karaca Ozkisacik, Department of Pediatric Surgery, Adnan Menderes Universitesi Cocuk Cerrahisi Anabilim Dali, Aydin, Turkey, Tel: +90 4441256-1455; E-mail: sozkisacik@gmail.com

\section{Case Report}

Volume 1 Issue 2

Received Date: August 16, 2017

Published Date: August 29, 2017

DOI: $10.23880 /$ mjccs- 16000108

\section{Abstract}

Introduction: Extrarenal Wilms tumor is extremely rare. The development of this tumor outside of the kidney parenchyma is rare. Case: 3 year old boy presented an abdominal pain. A mass was palpated anteriorly of the rectum on rectal examination.

Magnetic Resonans (MR) scan with intravenous (IV) contrast revealed a 47x43x23 millimeters, heterogeneous pelvic mass located rectovesical area. At laparotomy, tumor was fixed at the level of anterior recto sigmoid joint.

Pathologic diagnosis of the tumor was extrarenal Wilms tumor. There was no any leymphoid hypertrophy and metastasis. After complete tumor resection, tumor phase was accepted Stage 3 and chemotherapy and radiotherapy were administered according to the treatment protocol of the National Tumor Wilm's Study Group. A year after treatment, the patient has had no evidence of recurrence.

Conclusion: It is important to include extrarenal Wilms tumor in the differential diagnosis of children with intrathoracic or retroperitoneal tumors. Because some of these tumors are located in the pelvis, they may be incorrectly diagnosed preoperatively as a pelvic teratoma or ovarian cysts.

Keywords: Wilms Tumor; Rectosigmoid; Intrathoracic; Nephroblastoma; Abdominal Ultrasonography

\section{Introduction}

Wilms tumor, or nephroblastoma, is one of the most common solid malignant neoplasms in children and it is almost arising from the kidney [1]. The development of this tumor outside of the kidney parenchyma is rare. Most of these tumors located in the retroperitoneal space, but some tumors located elsewhere [2]. We present a 3-year old male with an extrarenal Wilms tumor located in the rectosigmoid joint in intraperitoneal space.

\section{Case}

A previously healthy 3 year old boy presented an abdominal pain. A mass was palpated anteriorly of the rectum on rectal examination. Other physical examination findings were normal. An abdominal ultrasonography (US) showed a mass which was $5 \times 3.5$ centimeters diameter, heterogenous, had smoot edges, located vesicorectal area. Magnetic Resonance (MR) scan with intravenous (IV) contrast revealed a $47 \times 43 \times 23$ millimeters, heterogeneous pelvic mass located 


\section{Medical Journal of Clinical Trials \& Case Studies}

rectovesical area. Others organs were evaluated normally. Laboratory tests were all within normal limits including serum $\alpha$-fetoprotein, $\beta$-human chorionic gonadotropin, and urinary catecholamines (vanillylmandelic acid and homovanillic acid).

At laparotomy, a tumor was located anterior of the rectum. Tumor was fixed at the level of anterior rectosigmoid joint (Figure 1). There was no ascites, lymphadenopathy or tumor invasion into surrounding tissues. The tumor was excised and submitted for pathologic diagnosis. On pathologic examination, the wellcircumscribed mass was $20 \mathrm{~g}$ and measured $4.4 \mathrm{~cm} \times 3.5$ $\mathrm{cm} \times 2.8 \mathrm{~cm}$. The tumor pathology was consistent with the diagnosis of extrarenal nephroblastoma.

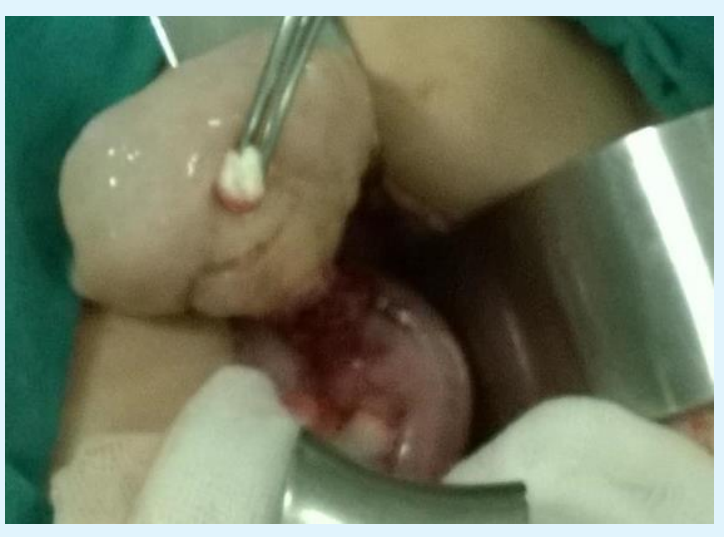

Figure 1: Intraoperative mass image.

After complete tumor resection, tumor phase was accepted Stage 3 and chemotherapy and radiotherapy was administered according to the treatment protocol of the Natioanl Tumor Wilm's Study Group. A year after treatment, the patient has had no evidence of recurrence.

\section{Discussion}

Intrarenal Wilms tumors are believed to originate from persistent metanephric blastema, but the histogenesis of extrarenal Wilms tumor (ERWT) is less clear. The general agreement is that the origin of ERWT parallels that of intrarenal Wilms tumor and arises from heterotopic metanephric blastema [3]. It is also believed that ERWT can originate from persistent mesonephric duct remnants [4].

Wilms tumors originating outside of the kidney are extremely rare. Because of the lack of specific tumor markers, preoperative diagnosis of extrarenal Wilms tumor is difficult. So the diagnosis of extrarenal Wilms tumor is almost made after resection of the tumor and pathologic evaluation of the specimen. According to the National Wilms Tumor Study (NWTS) the diagnosis of an extra renal Wilms tumor should fulfill three criteria. First, a primary tumor in the kidney should be excluded. Careful evaluation should be made, both radio graphically and intra operatively, to rule out a primary intra renal tumor with secondary extrarenal metastasis. We evaluated normally all of abdominal organs. Second, on pathologic evaluation the triphasic histology pattern of blastemal, epithelial, and primitive stroma characteristic of Wilms tumor should be present. And finally, there should be no evidence of teratoma or renal carcinoma on thorough examination of the whole tumor [3].

Extra renal Wilms tumor is an extremely rare malignant renal neoplasm. It is important to include extrarenal Wilms tumor in the differential diagnosis of children with intrathoracic or retroperitoneal tumors. Because some of these tumors are located in the pelvis, they may be incorrectly diagnosed preoperatively as a pelvic teratoma or ovarian cyst [5].

Following complete tumor excision, careful histologic evaluation will provide the correct diagnosis and permit selection of appropriate adjunctive treatment.

\section{References}

1. Rojas Y, Slater BJ, Braverman RM, Eldin KW, Thompson PA et al. (2013) Extrarenal Wilms tumor: a case report and review of the literature. J Pediatr Surg 48(6): E33-E35.

2. Iraniha S, Shen V, Kruppe CN, Downey EC (1999) Uterine cervical extrarenal Wilms tumor managed without hysterectomy. J Pediatr Hematol Oncol 21(6): 548-550.

3. Andrews PE, Kelalis PP, Haase GM (1992) Extrarenal Wilms' tumor: results of the National Wilms' Tumor Study. J Pediatr Surg 27(9): 1181-1184.

4. Orlowski JP, Levin HS, Dyment PG (1980) Intrascrotal Wilms' tumor developing in a heterotopic renal anlage of probable mesonephric origin. J Pediatr Surg 15(8): 679-682.

5. Nara KKTYM (2005) A case of extrarenal nephroblastoma. Nihonshounigekagakkaizassi 1 : 696.

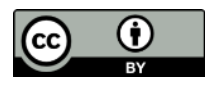

